\title{
Global dynamics of an SEIR epidemic model with discontinuous treatment
}

\author{
Tailei Zhang ${ }^{1}$, Ruini Kang ${ }^{1}$, Kai Wang ${ }^{2 *}$ and Junli Liu ${ }^{3}$
}

"Correspondence:

wangkaimath@sina.com

${ }^{2}$ Department of Medical

Engineering and Technology,

Xinjiang Medical University, Urumqi, 830011, P.R. China

Full list of author information is available at the end of the article

\begin{abstract}
We consider a susceptible-exposed-infected-removed (SEIR) epidemic model with discontinuous treatment strategies. The treatment rate has at most a finite number of jump discontinuities in every compact interval. By using Lyapunov theory for discontinuous differential equations and other techniques on non-smooth analysis, the basic reproductive number $\mathcal{R}_{0}$ is proved to be a sharp threshold value which completely determines the dynamics of the model. If $\mathcal{R}_{0} \leq 1$, then there only exists a disease-free equilibrium which is globally stable. If $\mathcal{R}_{0}>1$, the disease-free equilibrium becomes unstable and there exists a unique endemic equilibrium which is globally stable. The numerical simulations indicate that strengthening treatment measures after infective individuals reach some level is beneficial to disease control. Furthermore, we discuss that the disease will die out in a finite time, which is impossible for the corresponding SEIR model with continuous treatment.
\end{abstract}

Keywords: latent period; discontinuous treatment; epidemic dynamics; extinction of disease in finite time

\section{照 Springer}

\section{Introduction}

Infectious diseases can arise if the host's protective immune mechanisms are compromised and the organism inflicts damage on the host. It shows that the infectious disease can causes millions of deaths every year. Hence, how to prevent or slow down the transmission of infectious diseases is a very important problem. Many methods for control of infectious diseases are extensively applied, such as treatment, quarantine, isolation, immunity etc. To understand how to control and eradicate infectious disease is also one of the main goals of mathematical epidemiology. At the same time, well understanding for dynamic behaviors of infectious disease is a benefit for diseases controlling. Researchers have proposed many epidemic models to understand the mechanism of disease transmission (see [1-6] and the references therein).

Treatment plays a very important role in controlling the spread of diseases such as HIV/AIDS, tuberculosis, malaria, which are the top three single disease killers in the world. In recent years, some mathematical models incorporating treatment have been studied by many researchers (see [7-15] and the references therein). In [14], Wang and Ruan proposed an epidemic model to simulate the limited resources for the treatment of patients, which can occur because patients have to be hospitalized but there are limited beds in hospitals, or there is not enough medicine for treatments. In [15], Wang adopted

(c) 2015 Zhang et al. This article is distributed under the terms of the Creative Commons Attribution 4.0 International License (http://creativecommons.org/licenses/by/4.0/), which permits unrestricted use, distribution, and reproduction in any medium, provided you give appropriate credit to the original author(s) and the source, provide a link to the Creative Commons license, and indicate if changes were made. 
a constant treatment, which simulates a limited capacity for treatment. Note that a constant treatment is suitable when the number of infectives is large. Li et al. [10] constructed an SIR epidemic model with nonlinear incidence and treatment. The results show that a backward bifurcation occurs if the capacity is small and there exist bistable endemic equilibria if the capacity is low. Recently, Guo et al. considered an SIR epidemic model with discontinuous treatment strategies. The results show that discontinuous treatment strategies would be superior to continuous ones [11]. On the other hand, there are a lot of infectious diseases (e.g. TB, HIV/AIDS, malaria, SARS etc.) which have latent periods. That is, a susceptible individual first goes through a latent period after infection before becoming infectious. When we use mathematical models to analyze the above-mentioned diseases, omitting the latent period will lead to some inaccurate results on their transmission law. Under this circumstance, the epidemic models with latent periods play a very important role in epidemiology. Some models with latent periods can be called SEI, SEIS, SEIR or SEIRS type, respectively [16-18]. In [16], the authors studied the global dynamics of an SEIR model with this saturating contact rate.

This work was intended as an attempt to motivate [11] where infective individuals are removed from the infective class due to the treatment at a discontinuous rate. At the same time, the latent period is considered in the model. Due to this discontinuous treatment strategy, the resulting model is a discontinuous system. Some non-smooth analysis techniques [19] are used for this system. The paper is organized as follows. In the next section, we will construct the model and introduce the main assumptions for a discontinuous treatment function. In Section 3, positivity and boundedness of the solution in the sense of Filippov for the model will be clearly discussed. We obtain the existence of possible equilibria, the basic reproductive number, and the stability of equilibria in Section 4. In Section 5, we summarize our main results and discuss the possibility of the extinction of the infectives in a finite time.

\section{Model and preliminaries}

We consider a model with state variables $S, E, I$, and $R$, which represent the number of susceptible, exposed, infected, and removed individuals, respectively. The infected individuals will be given a discontinuous treatment. Consider the following SEIR model with discontinuous treatment:

$$
\left\{\begin{array}{l}
\frac{\mathrm{d} S}{\mathrm{~d} t}=\Lambda-\mu S-\beta S I, \\
\frac{\mathrm{d} E}{\mathrm{~d} t}=\beta S I-(\mu+\varepsilon) E, \\
\frac{\mathrm{d} I}{\mathrm{~d} t}=\varepsilon E-(\mu+\alpha+\gamma) I-h(I), \\
\frac{\mathrm{d} R}{\mathrm{~d} t}=\gamma I+h(I)-\mu R,
\end{array}\right.
$$

where $\Lambda$ is the growth rate of population, $\mu$ is the natural mortality rate, $\beta$ is the contact rate, $\alpha$ is the death rate induced by disease, $\varepsilon$ and $\gamma$ are the rates of naturally leaving the latent stage and infected stage, respectively. The function $h(I)=\phi(I) I$ denotes the treatment rate. $\phi(I)$ satisfies the following assumptions. Obviously, the treatment rate should be nondecreasing as the number of infectious individuals is increasing. The following assumption will be needed throughout the paper.

$\left(\mathrm{H}_{1}\right) \phi:[0, \infty) \rightarrow[0, \infty)$ is nondecreasing and has at most a finite number of jump discontinuities in every compact interval. There is no loss of generality in assuming $\phi$ 
is continuous at $I=0$, otherwise we define $\phi(0)$ to be $\phi\left(0^{+}\right)$. Here $\phi\left(0^{+}\right)$denotes the right limit of $\phi(I)$ as $I \rightarrow 0^{+}$.

Since the variable $R$ does not appear in the first three equations of model (2.1), we only need to study the first three equations of model (2.1), thereby lowering the order of the system to be studied, i.e.,

$$
\left\{\begin{array}{l}
\frac{\mathrm{d} S}{\mathrm{~d} t}=\Lambda-\mu S-\beta S I, \\
\frac{\mathrm{d} E}{\mathrm{~d} t}=\beta S I-(\mu+\varepsilon) E, \\
\frac{\mathrm{d} I}{\mathrm{~d} t}=\varepsilon E-(\mu+\alpha+\gamma) I-h(I) .
\end{array}\right.
$$

By the definition of solutions for differential equations with discontinuous right-hand sides in $[19,20]$, we call $(S(t), E(t), I(t))$ a solution with initial condition

$$
(S(0), E(0), I(0))=\left(S_{0}, E_{0}, I_{0}\right), \quad S_{0}, E_{0}, I_{0} \geq 0
$$

of model (2.2) on [0,T), $0<T \leq \infty$, if it is absolutely continuous on any compact subinterval of $[0, T)$, and almost everywhere on $[0, T)$ (abbreviated to 'a.e. on $\left.[0, T)^{\prime}\right)$ satisfies the following differential inclusion:

$$
\left\{\begin{array}{l}
\frac{\mathrm{d} S}{\mathrm{~d} t}=\Lambda-\mu S-\beta S I, \\
\frac{\mathrm{d} E}{\mathrm{~d} t}=\beta S I-(\mu+\varepsilon) E, \\
\frac{\mathrm{d} I}{\mathrm{~d} t} \in \varepsilon E-(\mu+\alpha+\gamma) I-\overline{\mathrm{co}}[h(I)],
\end{array}\right.
$$

where $\overline{\mathrm{co}}[h(I)]=[h(I-0), h(I+0)]$. Here, $h(I-0)$ and $h(I+0)$ denote the left limit and the right limit of the function $h(I)$ at $I$, respectively.

From $\left(\mathrm{H}_{1}\right)$, it is clear that the set map

$$
(S, E, I) \longmapsto(\Lambda-\mu S-\beta S I, \beta S I-(\mu+\varepsilon) E, \varepsilon E-(\mu+\alpha+\gamma) I-\overline{\mathrm{co}}[h(I)])
$$

is an upper semi-continuous set-valued map with non-empty compact convex values. By the measurable selection theorem ([19], p.17, Theorem 2.2.5), if $(S(t), E(t), I(t))$ is a solution of model (2.2) on $[0, T)$, then there is a measurable function $m(t) \in \overline{\mathrm{co}}[h(I(t))]$ such that

$$
\left\{\begin{array}{l}
\frac{\mathrm{d} S}{\mathrm{~d} t}=\Lambda-\mu S-\beta S I, \\
\frac{\mathrm{d} E}{\mathrm{~d} t}=\beta S I-(\mu+\varepsilon) E, \\
\frac{\mathrm{d} I}{\mathrm{~d} t}=\varepsilon E-(\mu+\alpha+\gamma) I-m(t),
\end{array} \quad \text { a.e. on }[0, T) .\right.
$$

\section{Positivity and boundedness}

In this section, we will prove that the solutions exist on $[0,+\infty)$ and are nonnegative. The main result is as follows.

Theorem 3.1 Suppose that assumption $\left(\mathrm{H}_{1}\right)$ holds and let $(S(t), E(t), I(t))$ be the solution with initial condition (2.3) of model (2.2) on $[0, T)$. Then $(S(t), E(t), I(t))$ is nonnegative and bounded on $[0, T)$. 
Proof By the definition of a solution of (2.2) in the sense of Filippov, $(S(t), E(t), I(t))$ must be a solution to differential inclusion (2.4). From the first equation of (2.4), we have

$$
S(t)=\left[S_{0}+\int_{0}^{t} \Lambda \exp \left(\int_{0}^{u} \mu+\beta I(\rho) \mathrm{d} \rho\right) \mathrm{d} u\right] \exp \left(-\int_{0}^{t} \mu+\beta I(\rho) \mathrm{d} \rho\right)>0
$$

for all $t \in(0, T)$.

According to $\left(\mathrm{H}_{1}\right)$, we have $\overline{\mathrm{co}}[h(0)]=\{0\}$ and $h(I)$ is continuous at $I=0$. Combining the continuity of $\phi$ at $I=0$, it may be concluded that there exists a positive constant $\delta$ such that $\phi(I)$ is continuous as $|I|<\delta$. For this reason, the differential inclusion (2.4) becomes the following system of differential equations as $|I|<\delta$ :

$$
\left\{\begin{array}{l}
\frac{\mathrm{d} E}{\mathrm{~d} t}=\beta S I-(\mu+\varepsilon) E \\
\frac{\mathrm{d} I}{\mathrm{~d} t}=\varepsilon E-(\mu+\alpha+\gamma+\phi(I)) I .
\end{array}\right.
$$

We divide this into four cases to discuss the positivity of the solutions for (2.4).

(i) $E_{0}=I_{0}=0$.

From (3.2), we see that $E(t)=I(t)=0$ for all $t \in[0, T)$.

(ii) $E_{0}>0, I_{0}=0$.

By the continuity of $E(t)$ at $t=0$ and $\left.\frac{\mathrm{d} I}{\mathrm{~d} t}\right|_{t=0}=\varepsilon E_{0}>0$, we conclude $E(t)>0$ and $I(t)>0$ for all $t \in(0, T)$. If it is not true, then we can set

$$
t_{1}=\inf \{t: E(t)=0 \text { or } I(t)=0\} \in(0, T) .
$$

If $E\left(t_{1}\right)=0$, then from $\frac{\mathrm{d} E}{\mathrm{~d} t} \geq-(\mu+\varepsilon) E$ for $0 \leq t \leq t_{1}$ we deduce that $E\left(t_{1}\right) \geq E_{0} \exp (-(\mu+$ $\left.\varepsilon) t_{1}\right)>0$. This is a contradiction. If $I\left(t_{1}\right)=0$, then there is a $\theta$ such that $t_{1}-\theta>0$ and $0<I(t)<\delta$ on $\left[t_{1}-\theta, t_{1}\right)$. Therefore, the second equation of (3.2) implies

$$
\frac{\mathrm{d} I}{\mathrm{~d} t} \geq-(\mu+\alpha+\gamma+\phi(I)) I
$$

This gives

$$
I\left(t_{1}\right) \geq I\left(t_{1}-\theta\right) \exp \left(-\int_{t_{1}-\theta}^{t_{1}}(\mu+\alpha+\gamma+\phi(I(\xi))) \mathrm{d} \xi\right)>0 .
$$

This is also a contradiction. Hence, $E(t)$ and $I(t)$ are positive for all $t \in(0, T)$. The same conclusion can be drawn for the following cases.

(iii) $E_{0}=0, I_{0}>0$.

(iv) $E_{0}>0, I_{0}>0$.

Our next goal is to prove the boundedness of the solutions of model (2.4).

Under the basis of above proof, adding the three equations of (2.4) yields

$$
\frac{\mathrm{d}(S+E+I)}{\mathrm{d} t} \in \Lambda-\mu(S+E+I)-(\alpha+\gamma) I-\overline{\mathrm{co}}[h(I)] .
$$

Fix any $v \in \overline{\mathrm{co}}[h(I)]$. When $S+E+I>\frac{\Lambda}{\mu}$, we must have

$$
\Lambda-\mu(S+E+I)-(\alpha+\gamma) I-v<0 .
$$


For this reason, we have $0 \leq S+E+I \leq \max \left\{\frac{\Lambda}{\mu}, S_{0}+E_{0}+I_{0}\right\}$, that is, $(S(t), E(t), I(t))$ is bounded on $[0, T)$. By the boundedness and the continuation theorem, we concluded that the solution $(S(t), E(t), I(t))$ exists on $[0,+\infty)$, namely, the solution is global existence. This completes the proof.

Remark 3.1 For any solution of (2.4) with nonnegative initial value (2.3), we have the following detailed statements, (i), (ii), and (iii).

(i) The solution $(S(t), E(t), I(t))$ of (2.4) exists on $[0,+\infty)$ and $S(t)>0(t>0), E(t) \geq 0$ $(t>0), I(t) \geq 0(t>0)$.

(ii) If $E(0)=0$ and $I(0)=0$, then the solution $(S(t), E(t), I(t))$ of $(2.4)$ exists on $[0,+\infty)$, $S(t)>0(t>0), E(t) \equiv 0(t \geq 0), I(t) \equiv 0(t \geq 0)$.

(iii) If one of $E(0)$ and $I(0)$ is greater than zero, then the solution $(S(t), E(t), I(t))$ of $(2.4)$ exists on $[0,+\infty)$ and $S(t)>0(t>0), E(t)>0(t>0), I(t)>0(t>0)$.

\section{Stability of equilibria}

In this section, we show the stability of the equilibria for model (2.2). We first discuss the existence of the equilibria as follows.

An equilibrium of model (2.2) is, by definition, a constant solution of $(2.2),(S(t), E(t)$, $I(t))=\left(S^{*}, E^{*}, I^{*}\right)$, where $\left(S^{*}, E^{*}, I^{*}\right)$ satisfies the following system:

$$
\left\{\begin{array}{l}
0=\Lambda-\mu S^{*}-\beta S^{*} I^{*} \\
0=\beta S^{*} I^{*}-(\mu+\varepsilon) E^{*} \\
0 \in \varepsilon E^{*}-(\mu+\alpha+\gamma) I^{*}-\overline{\operatorname{co}}\left[h\left(I^{*}\right)\right] .
\end{array}\right.
$$

Since $h(0)=0$, it follows that there always exists a disease-free equilibrium $P_{0}\left(\frac{\Lambda}{\mu}, 0,0\right)$. Next, we will consider the existence of an endemic equilibrium. By the first and second equations of (4.1), we conclude that

$$
S^{*}=\frac{\Lambda}{\mu+\beta I^{*}}, \quad E^{*}=\frac{\beta \Lambda I^{*}}{\left(\mu+\beta I^{*}\right)(\mu+\varepsilon)} .
$$

Substituting (4.2) into the third inclusion of (4.1) yields

$$
\frac{\varepsilon \beta \Lambda}{(\mu+\varepsilon)\left(\mu+\beta I^{*}\right)}-(\mu+\alpha+\gamma) \in \overline{\mathrm{co}}\left[\phi\left(I^{*}\right)\right]=\left[\phi\left(I^{*}-0\right), \phi\left(I^{*}+0\right)\right] .
$$

Write

$$
g\left(I^{*}\right)=\frac{\varepsilon \beta \Lambda}{(\mu+\varepsilon)\left(\mu+\beta I^{*}\right)}-(\mu+\alpha+\gamma)
$$

and let

$$
\mathcal{R}_{0}=\frac{\varepsilon \beta \Lambda}{\mu(\mu+\varepsilon)(\mu+\alpha+\gamma+\phi(0))}
$$

We next claim that $\mathcal{R}_{0}$ is the basic reproductive number for the model (2.2) which will determine the existence of an endemic equilibrium. 
Theorem 4.1 Suppose that assumption $\left(\mathrm{H}_{1}\right)$ holds. If $\mathcal{R}_{0} \leq 1$, then there only exists a disease-free equilibrium $P_{0}\left(\frac{\Lambda}{\mu}, 0,0\right)$. If $\mathcal{R}_{0}>1$, then there exists a unique positive endemic equilibrium $P^{*}\left(S^{*}, E^{*}, I^{*}\right)$ except $P_{0}$.

Proof As $\mathcal{R}_{0} \leq 1$, we have $g(0) \leq \phi(0)$. Since $g(I)$ is nonincreasing on $I$ and $\phi(I)$ is nondecreasing on $I$. For this reason, the inclusion (4.3) is only valid at $I=0$. Hence, the model (2.2) has a unique disease-free equilibrium as long as $\mathcal{R}_{0} \leq 1$.

If $\mathcal{R}_{0}>1$, then $g(0)>\phi(0) \geq 0$. At the same time, the inequality

$$
I \geq \frac{1}{\beta}\left[\frac{\varepsilon \beta \Lambda}{(\mu+\varepsilon)(\mu+\alpha+\gamma)}-\mu\right]
$$

implies $g(I) \leq 0$. Therefore, the set

$$
\Gamma=\{I: g(I) \geq \phi(I+0), I>0\}
$$

is bounded and non-empty. We can write

$$
I^{*}=\sup \{I: g(I) \geq \phi(I+0), I>0\} .
$$

It follows easily that

$$
g\left(I^{*}\right) \geq \phi\left(I^{*}-0\right) \quad \text { and } \quad 0<I^{*} \leq \frac{1}{\beta}\left[\frac{\varepsilon \beta \Lambda}{(\mu+\varepsilon)(\mu+\alpha+\gamma)}-\mu\right]
$$

We claim $g\left(I^{*}\right) \in\left[\phi\left(I^{*}-0\right), \phi\left(I^{*}+0\right)\right]$. Suppose, contrary to our claim, that

$$
g\left(I^{*}\right)>\phi\left(I^{*}+0\right)=\lim _{I \rightarrow I^{*}+0} \phi(I)
$$

From $\left(\mathrm{H}_{1}\right)$, there exists a $\delta>0$ such that

$$
g\left(I^{*}+\delta\right)>\phi\left(I^{*}+\delta\right)=\phi\left(I^{*}+\delta+0\right) .
$$

This contradicts the definition of $I^{*}$. Thus, we have $g\left(I^{*}\right) \in\left[\phi\left(I^{*}-0\right), \phi\left(I^{*}+0\right)\right]$. That is to say, $I^{*}$ is a positive solution of the inclusion (4.3). We proceed to show that $I^{*}$ is the only one positive solution of the inclusion (4.3). If the inclusion (4.3) has another positive solution $I^{* *}$, then there must exist two numbers

$$
\eta^{*} \in \overline{\mathrm{co}}\left[\phi\left(I^{*}\right)\right] \quad \text { and } \quad \eta^{* *} \in \overline{\mathrm{co}}\left[\phi\left(I^{* *}\right)\right]
$$

which satisfy

$$
\frac{\varepsilon \beta \Lambda}{(\mu+\varepsilon)\left(\mu+\beta I^{*}\right)}-(\mu+\alpha+\gamma)=\eta^{*}
$$

and

$$
\frac{\varepsilon \beta \Lambda}{(\mu+\varepsilon)\left(\mu+\beta I^{* *}\right)}-(\mu+\alpha+\gamma)=\eta^{* *} .
$$


Subtracting (4.10) from (4.9) gives

$$
\frac{\varepsilon \beta^{2} \Lambda\left(I^{* *}-I^{*}\right)}{(\mu+\varepsilon)\left(\mu+\beta I^{*}\right)\left(\mu+\beta I^{* *}\right)}=\eta^{*}-\eta^{* *}
$$

which implies

$$
\frac{\eta^{*}-\eta^{* *}}{I^{*}-I^{* *}}<0
$$

This is a contradiction. Hence, $I^{*}$ is the unique positive solution of the inclusion (4.3). Combining it with (4.2), we conclude that $\left(S^{*}, E^{*}, I^{*}\right)$ is the unique endemic equilibrium of (2.2). The proof is completed.

In the next part, we show the global stability of the disease-free equilibrium and the endemic equilibrium. We do this in several steps. We first prove their local stability as follows.

Theorem 4.2 Assume $\left(\mathrm{H}_{1}\right)$ holds. The disease-free equilibrium $P_{0}$ is locally asymptotically stable if $\mathcal{R}_{0}<1$, and is unstable if $\mathcal{R}_{0}>1$.

Proof We analyze the stability of the disease-free equilibrium by investigating the eigenvalues of the Jacobian matrix of model (2.2) at $P_{0}$. The matrix is

$$
J\left(P_{0}\right)=\left(\begin{array}{ccc}
-\mu & 0 & -\beta \frac{\Lambda}{\mu} \\
0 & -(\mu+\varepsilon) & \beta \frac{\Lambda}{\mu} \\
0 & \varepsilon & -(\mu+\alpha+\gamma)-\phi(0)
\end{array}\right) .
$$

The characteristic equation of $J\left(P_{0}\right)$ is

$$
\begin{gathered}
(\lambda+\mu)\left[\lambda^{2}+(\mu+\varepsilon+\mu+\alpha+\gamma+\phi(0)) \lambda\right. \\
\left.\quad+(\mu+\varepsilon)(\mu+\alpha+\gamma+\phi(0))-\frac{\varepsilon \beta \Lambda}{\mu}\right]=0 .
\end{gathered}
$$

Equation (4.13) has three roots $\lambda_{1}=-\mu<0, \lambda_{2}$, and $\lambda_{3}$, where

$$
\begin{aligned}
& \lambda_{2}+\lambda_{3}=-(\mu+\varepsilon+\mu+\alpha+\gamma+\phi(0))<0, \\
& \lambda_{2} \lambda_{3}=(\mu+\varepsilon)(\mu+\alpha+\gamma+\phi(0))\left(1-\mathcal{R}_{0}\right) .
\end{aligned}
$$

From (4.14), it is easily seen that both the real parts of $\lambda_{2}$ and of $\lambda_{3}$ are negative when $\mathcal{R}_{0}<1$. When $\mathcal{R}_{0}>1$, one of $\lambda_{2}$ and $\lambda_{3}$ is a number with a positive real part. Thus the disease-free equilibrium is locally asymptotically stable if $\mathcal{R}_{0}<1$ and unstable if $\mathcal{R}_{0}>1$. Thus, these results establish the theorem.

We have shown that there exists a positive endemic equilibrium if and only if $\mathcal{R}_{0}>1$ in Theorem 4.1. Here, we will establish its local stability. 
Theorem 4.3 Suppose that assumption $\left(\mathrm{H}_{1}\right)$ holds. If $\mathcal{R}_{0}>1$, the endemic equilibrium $P^{*}$ of the system (2.2) is locally asymptotically stable.

Proof The Jacobian matrix of (2.2) at the endemic equilibrium $P^{*}=\left(S^{*}, E^{*}, I^{*}\right)$ is

$$
J\left(P^{*}\right)=\left(\begin{array}{ccc}
-\mu-\beta I^{*} & 0 & -\beta S^{*} \\
\beta I^{*} & -(\mu+\varepsilon) & \beta S^{*} \\
0 & \varepsilon & -(\mu+\alpha+\gamma)-\phi\left(I^{*}\right)-\phi^{\prime}\left(I^{*}\right) I^{*}
\end{array}\right) .
$$

Replacing $-(\mu+\alpha+\gamma)-\phi\left(I^{*}\right)$ by $\varepsilon \beta S^{*} /(\mu+\varepsilon)$ gives

$$
J\left(P^{*}\right)=\left(\begin{array}{ccc}
-\mu-\beta I^{*} & 0 & -\beta S^{*} \\
\beta I^{*} & -(\mu+\varepsilon) & \beta S^{*} \\
0 & \varepsilon & -\frac{\varepsilon \beta S^{*}}{\mu+\varepsilon}-\phi^{\prime}\left(I^{*}\right) I^{*}
\end{array}\right) .
$$

The characteristic equation of $J\left(P^{*}\right)$ is

$$
\lambda^{3}+a_{1} \lambda^{2}+a_{2} \lambda+a_{3}=0
$$

where

$$
\begin{aligned}
& a_{1}=\mu+\beta I^{*}+\mu+\varepsilon+\frac{\varepsilon \beta S^{*}}{\mu+\varepsilon}+\phi^{\prime}\left(I^{*}\right) I^{*} \\
& a_{2}=\left(\mu+\beta I^{*}\right)\left(\mu+\varepsilon+\frac{\varepsilon \beta S^{*}}{\mu+\varepsilon}+\phi^{\prime}\left(I^{*}\right) I^{*}\right)+(\mu+\varepsilon) \phi^{\prime}\left(I^{*}\right) I^{*}, \\
& a_{3}=\left(\mu+\beta I^{*}\right)(\mu+\varepsilon) \phi^{\prime}\left(I^{*}\right) I^{*}+\varepsilon \beta^{2} S^{*} I^{*}
\end{aligned}
$$

Since $\phi$ is nondecreasing, $\phi^{\prime}\left(I^{*}\right) \geq 0$. This implies $a_{1}>0, a_{2}>0, a_{3}>0$.

Then

$$
\begin{aligned}
a_{1} a_{2}-a_{3}= & {\left[\mu+\beta I^{*}+\mu+\varepsilon+\frac{\varepsilon \beta S^{*}}{\mu+\varepsilon}+\phi^{\prime}\left(I^{*}\right) I^{*}\right] } \\
& \times\left[\left(\mu+\beta I^{*}\right)\left(\mu+\varepsilon+\frac{\varepsilon \beta S^{*}}{\mu+\varepsilon}+\phi^{\prime}\left(I^{*}\right) I^{*}\right)+(\mu+\varepsilon) \phi^{\prime}\left(I^{*}\right) I^{*}\right] \\
& -\left(\mu+\beta I^{*}\right)(\mu+\varepsilon) \phi^{\prime}\left(I^{*}\right) I^{*}+\varepsilon \beta^{2} S^{*} I^{*} \\
> & (\mu+\varepsilon)\left(\mu+\beta I^{*}\right)\left(\frac{\varepsilon \beta S^{*}}{\mu+\varepsilon}+\phi^{\prime}\left(I^{*}\right) I^{*}\right) \\
& -\left(\mu+\beta I^{*}\right)(\mu+\varepsilon) \phi^{\prime}\left(I^{*}\right) I^{*}-\varepsilon \beta^{2} S^{*} I^{*} \\
> & 0 .
\end{aligned}
$$

Hence, all of the Routh-Hurwitz criteria are satisfied. Thus it follows that the endemic equilibrium $P^{*}$ of $(2.2)$, which exists if $\mathcal{R}_{0}>1$, is always locally asymptotically stable. The proof is completed.

We next prove global stability of the disease-free equilibrium and endemic equilibrium. We need to use the LaSalle-type invariance principle for the differential inclusion (Theorem 3 in [21]) to prove their global stability. 
Let $x=S-\frac{\Lambda}{\mu}$. We obtain the following system analogous to (2.4):

$$
\left\{\begin{array}{l}
\frac{\mathrm{d} x}{\mathrm{~d} t}=-\mu x-\beta x I-\beta \frac{\Lambda}{\mu} I \\
\frac{\mathrm{d} E}{\mathrm{~d} t}=\beta x I+\beta \frac{\Lambda}{\mu} I-(\mu+\varepsilon) E \\
\frac{\mathrm{d} I}{\mathrm{~d} t} \in \varepsilon E-(\mu+\alpha+\gamma) I-\overline{\mathrm{c}}[\phi(I)] I .
\end{array}\right.
$$

Set

$$
V_{1}(x, E, I)=\frac{x^{2}}{2}+\frac{\Lambda}{\mu} E+\frac{\Lambda}{\mu} \frac{\mu+\varepsilon}{\varepsilon} I
$$

and

$$
\mathbf{G}(x, E, I)=\left(\begin{array}{c}
-\mu x-\beta x I-\beta \frac{\Lambda}{\mu} I \\
\beta x I+\beta \frac{\Lambda}{\mu} I-(\mu+\varepsilon) E \\
\varepsilon E-(\mu+\alpha+\gamma) I-\overline{c o}[\phi(I)] I
\end{array}\right) .
$$

For any $\mathbf{v}=\left(v_{1}, v_{2}, v_{3}\right)^{T} \in \mathbf{G}(x, E, I)$, there exists an $\eta(t) \in \overline{\mathrm{co}}[\phi(I)]$ such that

$$
\mathbf{v}=\left(\begin{array}{c}
-\mu x-\beta x I-\beta \frac{\Lambda}{\mu} I \\
\beta x I+\beta \frac{\Lambda}{\mu} I-(\mu+\varepsilon) E \\
\varepsilon E-(\mu+\alpha+\gamma) I-\eta(t) I
\end{array}\right) .
$$

Hence,

$$
\begin{aligned}
\nabla V_{1}(x, E, I) \cdot \mathbf{v}= & \left(x, \frac{\Lambda}{\mu}, \frac{\Lambda(\mu+\varepsilon)}{\mu \varepsilon}\right)\left(\begin{array}{c}
-\mu x-\beta x I-\beta \frac{\Lambda}{\mu} I \\
\beta x I+\beta \frac{\Lambda}{\mu} I-(\mu+\varepsilon) E \\
\varepsilon E-(\mu+\alpha+\gamma) I-\eta(t) I
\end{array}\right) \\
= & -\mu x^{2}-\beta x^{2} I \\
& +\frac{\Lambda(\mu+\varepsilon)}{\mu \varepsilon}\left(\beta \frac{\Lambda}{\mu} \frac{\varepsilon}{\mu+\varepsilon}-(\mu+\alpha+\gamma+\eta(t))\right) I .
\end{aligned}
$$

When $\mathcal{R}_{0} \leq 1$, the nondecreasing of $\phi$ implies

$$
\beta \frac{\Lambda}{\mu} \frac{\varepsilon}{\mu+\varepsilon}-(\mu+\alpha+\gamma+\eta(t)) \leq \beta \frac{\Lambda}{\mu} \frac{\varepsilon}{\mu+\varepsilon}-(\mu+\alpha+\gamma+\phi(0)) \leq 0 .
$$

It shows that $V_{1}$ is a Lyapunov function of (4.17).

Furthermore, when $\mathcal{R}_{0}<1$, we have

$$
\begin{aligned}
\mathcal{Z}_{V_{1}} & \triangleq\left\{(x, E, I) \in \mathbb{R}^{3}: \nabla V_{1}(x, E, I) \cdot \mathbf{v}=0, \mathbf{v} \in \mathbf{G}(x, E, I)\right\} \\
& =\{(0, E, 0): E \geq 0\} .
\end{aligned}
$$

When $I=0$, we have $\frac{\mathrm{d} E}{\mathrm{~d} t}=-(\mu+\varepsilon) E$, which implies $\lim _{t \rightarrow \infty} E(t)=0$. For any $l>0$, we set

$$
V_{1}^{l} \triangleq\left\{(x, E, I) \in \mathbb{R}^{3}: V_{1}(x, E, I) \leq l\right\}
$$

Hence, the largest weakly invariant subset of $\overline{\mathcal{Z}_{V_{1}}} \cap V_{1}^{l}$ is the singleton $M=\{(0,0,0)\}$. 
When $\mathcal{R}_{0}=1$, we have

$$
\mathcal{Z}_{V_{1}}=\{(0, E, 0): E \geq 0\} \cup\{(0, E, I): \eta(t)=\phi(0), I>0\} .
$$

From the first equation of (4.17) and $x=0$, it may be concluded that $I=0$. Therefore, we see that the largest weakly invariant subset of $\overline{\mathcal{Z}_{V_{1}}} \cap V_{1}^{l}$ is also the singleton $M=\{(0,0,0)\}$. By the LaSalle-type invariance principle, the equilibrium $(0,0,0)$ of $(4.17)$ is globally asymptotically stable as $\mathcal{R}_{0} \leq 1$. Summarizing the above analysis, we obtain the following theorem.

Theorem 4.4 Suppose that assumption $\left(\mathrm{H}_{1}\right)$ holds. If $\mathcal{R}_{0} \leq 1$, then the disease-free equilibrium $P_{0}$ of $(2.4)$ is globally asymptotically stable.

The following theorem states the global stability of the endemic equilibrium $P^{*}$.

Theorem 4.5 Suppose that assumption $\left(\mathrm{H}_{1}\right)$ holds. If $\mathcal{R}_{0}>1$, then the endemic equilibrium $P^{*}$ of (2.4) is globally asymptotically stable.

Proof Let

$$
\begin{aligned}
V(S, E, I)= & \left(S-S^{*}-S^{*} \ln \frac{S}{S^{*}}\right)+\left(E-E^{*}-E^{*} \ln \frac{E}{E^{*}}\right) \\
& +\frac{\mu+\varepsilon}{\varepsilon}\left(I-I^{*}-I^{*} \ln \frac{I}{I^{*}}\right) .
\end{aligned}
$$

Write

$$
\eta^{*}=\frac{1}{I^{*}}\left(\varepsilon E^{*}-(\mu+\alpha+\gamma) I^{*}\right) \in \overline{\mathrm{co}}\left[\phi\left(I^{*}\right)\right]
$$

and

$$
\mathbf{H}(S, E, I)=\left(\begin{array}{c}
\Lambda-\mu S-\beta S I \\
\beta S I-(\mu+\varepsilon) E \\
\varepsilon E-(\mu+\alpha+\gamma) I-\overline{c o}[\phi(I)] I
\end{array}\right) .
$$

For any $\mathbf{v}=\left(v_{1}, v_{2}, v_{3}\right)^{T} \in \mathbf{H}(S, E, I)$, there exists an $\eta(t) \in \overline{\mathrm{co}}[\phi(I)]$ such that

$$
\mathbf{v}=\left(\begin{array}{c}
\Lambda-\mu S-\beta S I \\
\beta S I-(\mu+\varepsilon) E \\
\varepsilon E-(\mu+\alpha+\gamma) I-\eta(t) I
\end{array}\right) .
$$

The gradient of $V$ is given by

$$
\nabla V(S, E, I)=\left(1-\frac{S^{*}}{S}, 1-\frac{E^{*}}{E}, \frac{\mu+\varepsilon}{\varepsilon}\left(1-\frac{I^{*}}{I}\right)\right)^{T} .
$$


Hence

$$
\begin{aligned}
\nabla V(S, E, I) \cdot \mathbf{v}= & \left(1-\frac{S^{*}}{S}, 1-\frac{E^{*}}{E}, \frac{\mu+\varepsilon}{\varepsilon}\left(1-\frac{I^{*}}{I}\right)\right)\left(\begin{array}{c}
\Lambda-\mu S-\beta S I \\
\beta S I-(\mu+\varepsilon) E \\
\varepsilon E-(\mu+\alpha+\gamma) I-\eta(t) I
\end{array}\right) \\
= & \Lambda-\mu S-\frac{\Lambda S^{*}}{S}+\mu S^{*}+\beta S^{*} I-\beta S I \frac{E^{*}}{E}+(\mu+\varepsilon) E^{*} \\
& -(\mu+\varepsilon) \frac{E I^{*}}{I}-\frac{(\mu+\varepsilon)(\mu+\alpha+\gamma+\eta(t))}{\varepsilon}\left(I-I^{*}\right) \\
= & \mu S^{*}\left(2-\frac{S}{S^{*}}-\frac{S^{*}}{S}\right)+\beta S^{*} I^{*}\left(3-\frac{S^{*}}{S}-\frac{S E^{*} I}{S^{*} E I^{*}}-\frac{E I^{*}}{E^{*} I}\right) \\
& +\frac{(\mu+\varepsilon)}{\varepsilon}\left(\eta^{*}-\eta(t)\right)\left(I-I^{*}\right) .
\end{aligned}
$$

The monotonicity of $\phi$ implies $\left(\eta^{*}-\eta(t)\right)\left(I-I^{*}\right) \leq 0$. Thus $\nabla V(S, E, I) \cdot \mathbf{v} \leq 0$. This shows that $V$ is a Lyapunov function of (2.4). Define

$$
\begin{aligned}
\mathcal{Z}_{V} & =\left\{(S, E, I) \in \mathbb{R}_{+}^{3}: \exists \mathbf{v} \in \mathbf{H}(S, E, I), \nabla V(S, E, I) \cdot \mathbf{v}=0\right\} \\
& =\left\{\left(S^{*}, E^{*}, I^{*}\right)\right\} \cup\left\{\left(S^{*}, E, I\right): \frac{E}{E^{*}}=\frac{I}{I^{*}}, \eta^{*}=\eta(t)\right\} .
\end{aligned}
$$

If $S=S^{*}$, then the first equation of (2.4) implies $I=I^{*}$. This gives $E=E^{*}$. Consequently, for any $l>0$, the largest weakly invariant subset of $\overline{\mathcal{Z}_{V}} \cap V^{l}$ of (2.4) is the singleton $\left\{\left(S^{*}, E^{*}, I^{*}\right)\right\}$. Here

$$
V^{l}=\left\{\left(S-S^{*}, E-E^{*}, I-I^{*}\right) \in \mathbb{R}^{3}: V(S, E, I) \leq l\right\} .
$$

Therefore, $P^{*}$ is globally asymptotically stable if $\mathcal{R}_{0}>1$. This completes the proof.

Remark 4.1 From Theorems 4.2-4.5, we can claim that the basic reproduction number $\mathcal{R}_{0}$ is a sharp threshold value and that the global dynamical behaviors of the system (2.4) and the outcome of the disease are completely determined. In other words, when $\mathcal{R}_{0} \leq 1$, the disease-free equilibrium $P_{0}$ is globally stable so that the disease goes to extinction, while if $\mathcal{R}_{0}>1$, the endemic equilibrium $P^{*}$ is globally stable so that the disease remains endemic.

\section{Discussion}

We have considered an SEIR epidemic model that incorporates the discontinuous treatment strategies. Unlike previous SEIR epidemic models, we are interested in finding the impact of the adoption of a discontinuous treatment function.

The basic reproductive number $\mathcal{R}_{0}$ is derived under some reasonable assumptions on the discontinuous treatment function. It is a sharp threshold parameter which completely determines the global dynamics of the model (2.4) and whether the disease goes to extinction or not. When $\mathcal{R}_{0} \leq 1$, the disease-free equilibrium is globally stable so that the disease always dies out, and when $\mathcal{R}_{0}>1$, the disease-free equilibrium is unstable while the endemic equilibrium emerges as the unique positive equilibrium and it is globally stable. 
For making the utility of the concepts more visibly apparent, we present numerical simulations of solutions of the model (2.1). We give a treatment function satisfying $\left(\mathrm{H}_{1}\right)$ as follows:

$$
h(I)= \begin{cases}c_{1} I, & I \leq I_{0} \\ c_{2} I, & I>I_{0}\end{cases}
$$

where $0 \leq c_{1}<c_{2}$. It can be used to describe the following case: when the infective individuals attain some threshold $I_{0}$, we need to strengthen the treatment rate.

Let $\Lambda=240, \mu=0.02, \alpha=0.01, \gamma=0.3, \beta=0.0002, \varepsilon=0.2, c_{1}=0, I_{0}=30$, one could easily see that $\mathcal{R}_{0}=6.6116>1$ by using (4.5). Figure 1 shows that the infective will go to an endemic level. In addition, the basic reproductive number $\mathcal{R}_{0}$ is independent of $c_{2}$, but the different values of $c_{2}$ can affect the stability level of the infective. That is to say, larger values of $c_{2}$ can lead to a lower stability level of the infective. It shows that the strengthening of the treatment rate after the number of infective individuals has increased to some high level is also a beneficial disease control. Let $\Lambda=240, \mu=0.02, \alpha=0.01, \gamma=0.3, \beta=0.000026$, $\varepsilon=0.2, c_{1}=0, I_{0}=30$, one easily sees that $\mathcal{R}_{0}=0.8595<1$ by using (4.5). We obtain Figure 2 .

It shows that the disease goes to extinction. This numerical verification supports Theorem 4.4. In addition, we find that different values of $c_{2}$ can affect the peak values of the infective. Figure 2 reflects that larger values of $c_{2}$ can reduce the peak values of the infective. Therefore, we can also prevent the spread of disease by increasing the treatment rate after the number infective individuals reach some high level. From the numerical simulations, strengthening the treatment rate after the infective individuals reach some level is also effective for disease control, even though we do not take any treatment measures at the initial time of the disease's outbreak.

For a system of ordinary differential equations (ODE) with continuous right-hand side, any solutions cannot attain an equilibrium in a finite time. But an ODE system with discontinuous right-hand side can have the possibility that a solution converges to an equilibrium in a finite time. Next, we will discuss that the solutions converge to the disease-free equilibrium. Hence, we must assume the treatment function $h(I)$ in model (2.1) is discontinuous at $I=0$. Here we give the following assumption.

$\left(\mathrm{H}_{2}\right) h(I):[0, \infty) \rightarrow[0, \infty)$ is nondecreasing and has at most a finite number of jump discontinuities in every compact interval. Furthermore, $h(0)=0$ and $h(I)$ is discontinuous at $I=0$.

Under the assumption $\left(\mathrm{H}_{2}\right)$, it is easily to see that $\left(\frac{\Lambda}{\mu}, 0,0\right)$ is the disease-free equilibrium of (2.4). Let $x=S-\frac{\Lambda}{\mu}$. Then (2.4) changes into

$$
\left\{\begin{array}{l}
\frac{\mathrm{d} x}{\mathrm{~d} t}=-\mu x-\beta x I-\beta \frac{\Lambda}{\mu} I, \\
\frac{\mathrm{d} E}{\mathrm{~d} t}=\beta x I+\beta \frac{\Lambda}{\mu} I-(\mu+\varepsilon) E, \\
\frac{\mathrm{d} I}{\mathrm{~d} t} \in \varepsilon E-(\mu+\alpha+\gamma) I-\overline{\mathrm{co}}[h(I)] .
\end{array}\right.
$$

From (2.5), there exists a measurable function $\eta(t) \in \overline{\mathrm{co}}[h(I)]$ such that

$$
\left\{\begin{array}{l}
\frac{\mathrm{d} x}{\mathrm{~d} t}=-\mu x-\beta x I-\beta \frac{\Lambda}{\mu} I, \\
\frac{\mathrm{d} E}{\mathrm{~d} t}=\beta x I+\beta \frac{\Lambda}{\mu} I-(\mu+\varepsilon) E, \quad \text { for a.e. } t \in[0, \infty) . \\
\frac{\mathrm{d} I}{\mathrm{~d} t}=\varepsilon E-(\mu+\alpha+\gamma) I-\eta(t) .
\end{array}\right.
$$



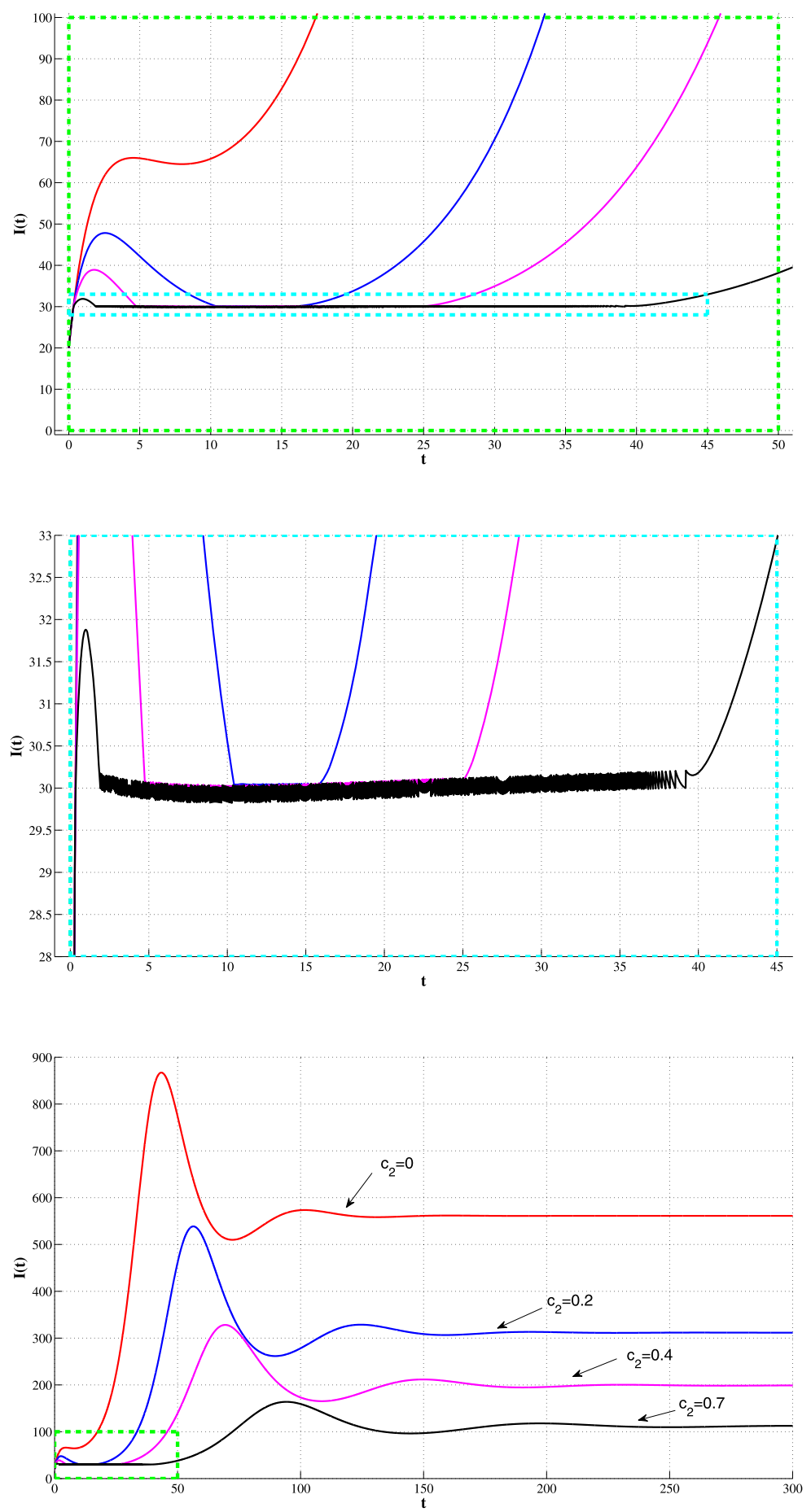

Figure 1 Time series plot of the infective population in symptomatic phase $(I)$ for $\mathcal{R}_{0}=6.6116>1$. The top two figures are the solution curves in the green box of the bottom figure. When the solution curves approach $I_{0}=30$, there appear many oscillations. 

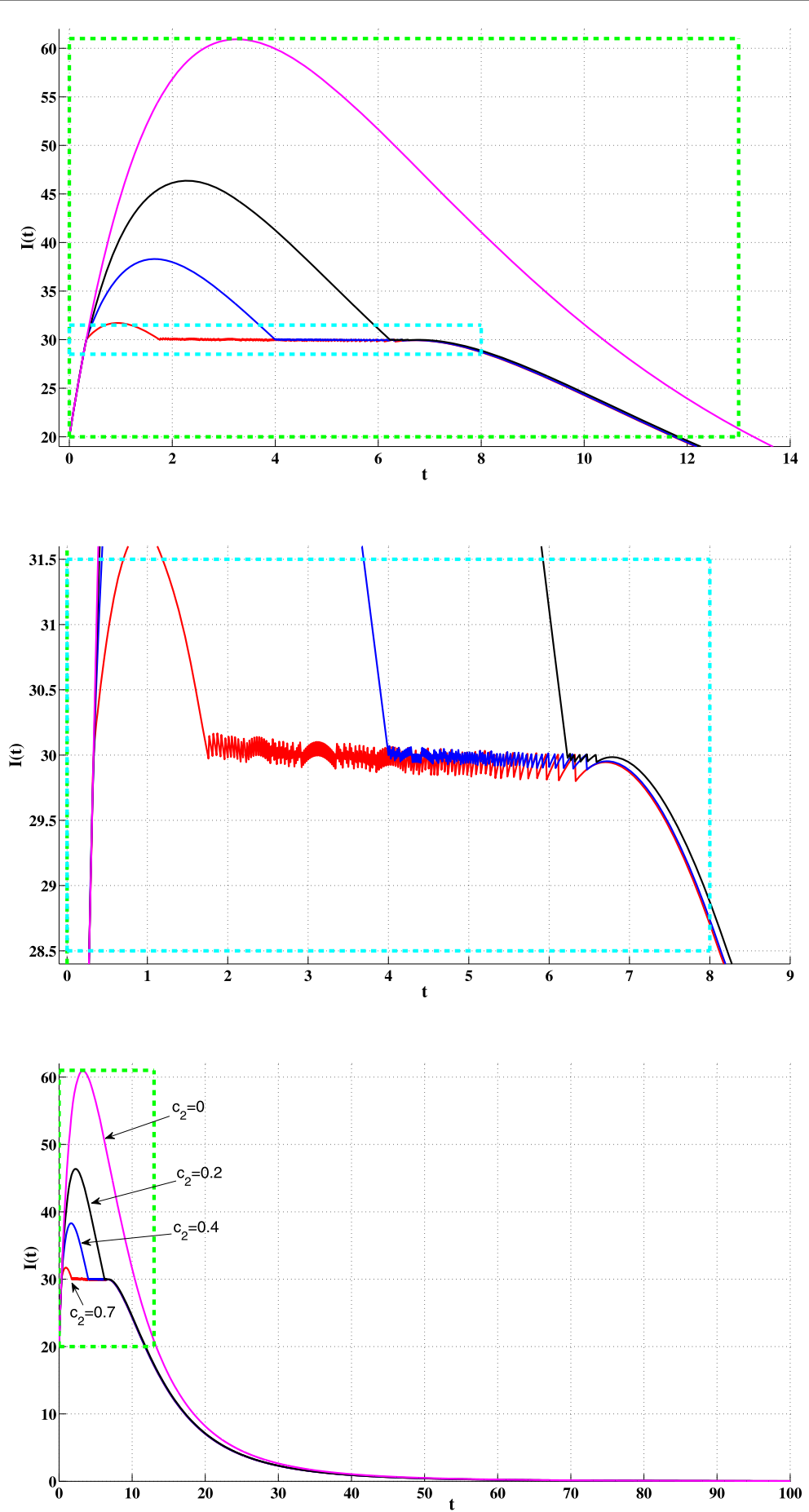

Figure 2 Time series plot of the infective population in symptomatic phase $(I)$ for $\mathcal{R}_{0}=0.8595<1$. The top two figures are the solution curves in the green box of the bottom figure. When the solution curves approach $I_{0}=30$, there appear many oscillations. 
Let $V_{1}(x, E, I)$ be the same Lyapunov function as (4.18). Its derivative along the solutions of (5.2) is

$$
\frac{\mathrm{d} V_{1}}{\mathrm{~d} t}=-\mu x^{2}-\beta x^{2} I+\frac{\Lambda}{\mu}\left(\beta \frac{\Lambda}{\mu}-\frac{(\mu+\varepsilon)(\mu+\alpha+\gamma)}{\varepsilon}\right) I-\frac{\Lambda}{\mu} \frac{\mu+\varepsilon}{\varepsilon} \eta(t) .
$$

By $\left(\mathrm{H}_{2}\right)$, we know that $\eta(t) \geq h\left(0^{+}\right)$. When $\frac{\varepsilon \beta \Lambda}{\mu(\mu+\varepsilon)(\mu+\alpha+\gamma)} \leq 1$, we have

$$
\frac{\mathrm{d} V_{1}}{\mathrm{~d} t} \leq-\frac{\Lambda}{\mu} \frac{\mu+\varepsilon}{\varepsilon} h\left(0^{+}\right)
$$

Integrating the differential inequality (5.3) from 0 to $t$ gives

$$
0 \leq V_{1}(x, E, I) \leq V_{1}(x(0), E(0), I(0))-\frac{\Lambda}{\mu} \frac{\mu+\varepsilon}{\varepsilon} h\left(0^{+}\right) t .
$$

Hence, when $t>t^{*}, V_{1}(x, E, I)=0$, this implies $(x, E, I)=(0,0,0)$, where

$$
t^{*}=\frac{\mu \varepsilon}{(\mu+\varepsilon) \Lambda h\left(0^{+}\right)}\left(\frac{\left(S(0)-\frac{\Lambda}{\mu}\right)^{2}}{2}+\frac{\Lambda}{\mu} E(0)+\frac{\Lambda}{\mu} \frac{\mu+\varepsilon}{\varepsilon} I(0)\right) .
$$

Consequently, every solution of $(2.4)(S(t), E(t), I(t))=\left(\frac{\Lambda}{\mu}, 0,0\right)$ for $t \geq t^{*}$.

From the form of $t^{*}$, we find that $t^{*}$ is increasing in the initial exposed and infective individuals but decreasing in the initial treatment rate $h\left(0^{+}\right)$. If we take more effective control measures for infectious diseases at the initial time of the diseases' spread, then the diseases go to extinction more quickly. The number $\frac{\varepsilon \beta \Lambda}{\mu(\mu+\varepsilon)(\mu+\alpha+\gamma)}$ is just the basic reproductive number of the SEIR model without treatment. The above analysis shows that the disease can go to extinction in a finite time under a discontinuous treatment strategy.

\section{Competing interests}

The authors declare that they have no competing interests.

\section{Authors' contributions}

TZ proposed the model and completed the main part of this manuscript, KW checked all the theorems and polished the language, JL enhanced the revised version, and RK revised the numerical simulation. All the authors read and approved the manuscript.

\section{Author details}

'School of Science, Chang'an University, Xi'an, 710064, P.R. China. ²Department of Medical Engineering and Technology, Xinjiang Medical University, Urumqi, 830011, P.R. China. ${ }^{3}$ School of Science, Xi'an Polytechnic University, Xi'an, 710048,

P.R. China.

\section{Acknowledgements}

This work was supported by the Fundamental Research Funds for the Central Universities (310812152002), the Project Supported by Natural Science Basic Research Plan in Shaanxi Province of China (No. 2014JQ1018) and National Natural Science Foundation of P.R. China (11201399, 11461073).

Received: 10 May 2015 Accepted: 11 November 2015 Published online: 25 November 2015

\section{References}

1. Anderson, RM, May, RM: Infectious Disease of Humans, Dynamical and Control. Oxford University Press, Oxford (1992)

2. Thieme, HR: Mathematics in Population Biology. Princeton University Press, Princeton (2003)

3. Ma, Z, Zhou, Y, Wang, W, Jin, Z: Mathematical Modelling and Research of Epidemic Dynamical Systems. Science Press, Beijing (2004) (in Chinese)

4. Hethcote, HW: The mathematics of infectious diseases. SIAM Rev. 42, 599-653 (2000)

5. Qin, W, Tang, S, Cheke, RA: Nonlinear pulse vaccination in an SIR epidemic model with resource limitation. Abstr. Appl. Anal. 2013, Article ID 670263 (2013) 
6. Xiao, Y, Zhao, T, Tang, S: Dynamics of an infectious disease with media/psychology induced non-smooth incidence. Math. Biosci. Eng. 10, 445-461 (2013)

7. Arino, J, Brauer, $F$, van den Driessche, $P$, Watmough, J, Wu, J: A model for influenza with vaccination and antiviral treatment. J. Theor. Biol. 253, 118-130 (2008)

8. Brauer, F: Epidemic models with heterogeneous mixing and treatment. Bull. Math. Biol. 70, 1869-1885 (2008)

9. $\mathrm{Hu}, \mathrm{Z}, \mathrm{Liu}, \mathrm{S}, \mathrm{Wang}, \mathrm{H}$ : Backward bifurcation of an epidemic model with standard incidence rate and treatment rate. Nonlinear Anal., Real World Appl. 9, 2302-2312 (2008)

10. Li, X, Li, W, Ghosh, M: Stability and bifurcation of an SIR epidemic model with nonlinear incidence and treatment. Appl. Math. Comput. 210, 141-150 (2009)

11. Guo, Z, Huang, L, Zou, X: Impact of discontinuous treatments on disease dynamics in an SIR epidemic model. Math. Biosci. Eng. 9, 97-110 (2012)

12. Xue, Y, Wang, J: Backward bifurcation of an epidemic model with infectious force in infected and immune period and treatment. Abstr. Appl. Anal. 2012, Article ID 647853 (2012)

13. Hussaini, $\mathrm{N}$, Winter, M: Travelling waves for an epidemic model with non-smooth treatment rates. J. Stat. Mech. 11, P11019 (2010)

14. Wang, W, Ruan, S: Bifurcations in an epidemic model with constant removal rate of the infectives. J. Math. Anal. Appl. 291, 775-793 (2004)

15. Wang, W: Backward bifurcation of an epidemic model with treatment. Math. Biosci. 201, 58-71 (2006)

16. Zhang, J, Ma, Z: Global dynamics of an SEIR epidemic model with saturating contact rate. Math. Biosci. 185, 15-32 (2003)

17. Zhang, T, Teng, Z: On a nonautonomous SEIRS model in epidemiology. Bull. Math. Biol. 69, 2537-2559 (2007)

18. Shu, H, Fan, D, Wei, J: Global stability of multi-group SEIR epidemic models with distributed delays and nonlinear transmission. Nonlinear Anal., Real World Appl. 13, 1581-1592 (2012)

19. Huang, L, Guo, Z, Wang, J: Theory and Applications of Differential Equations with Discontinuous Righthand Sides Science Press, Beijing (2011) (in Chinese)

20. Filippov, AF: Differential Equations with Discontinuous Righthand Sides. Mathematics and Its Applications (Soviet Series). Kluwer Academic, Boston (1988)

21. Baciotti, A, Ceragioli, F: Stability and stabilization of discontinuous systems and non-smooth Lyapunov function ESAIM Control Optim. Calc. Var. 4, 361-376 (1999)

\section{Submit your manuscript to a SpringerOpen ${ }^{\ominus}$ journal and benefit from:}

- Convenient online submission

Rigorous peer review

- Immediate publication on acceptance

- Open access: articles freely available online

- High visibility within the field

- Retaining the copyright to your article 